Article

\title{
Pre-Clinical Intravenous Serum Pharmacokinetics of Albumin Binding and Non-Half-Life Extended Nanobodies ${ }^{\circledR}{ }^{\dagger}$
}

\section{Sven Hoefman *, Ingrid Ottevaere, Judith Baumeister and Maria Laura Sargentini-Maier}

Ablynx N.V., Technologiepark 21, 9052 Zwijnaarde, Belgium; E-Mails: Ingrid.Ottevaere@ablynx.com (I.O.); Judith.Baumeister@ablynx.com (J.B.); Laura.Sargentini@ablynx.com (M.L.S.-M.)

$\dagger$ Nanobody is a registered trademark of Ablynx NV.

* Author to whom correspondence should be addressed; E-Mail: Sven.Hoefman@ablynx.com; Tel.: +32-(0)9-262-0000; Fax: +32-(0)9-262-0001.

Academic Editor: Friedrich Koch-Nolte

Received: 29 May 2015 / Accepted: 6 July 2015 / Published: 10 July 2015

\begin{abstract}
Nanobodies are antigen-binding, single variable domain proteins derived from naturally-occurring, heavy chain only antibodies. They are highly soluble, stable, and can be linked to build multi-specific formats. Several Nanobodies are currently in clinical development in different therapeutic areas, for both chronic and acute applications. For the former, prolonged exposure is achieved by half-life extending moieties that target endogenous albumin, while for the latter, non-half-life extended constructs are preferable. To demonstrate the general pharmacokinetic behavior of both formats, serum levels of seven intravenously administered Nanobodies were analyzed in cynomolgus monkeys, mice or rabbits. In monkeys, the total clearance of a monomeric irrelevant Nanobody was rapid $(2.0 \mathrm{~mL} /(\mathrm{min} * \mathrm{~kg}))$ and approximated the species glomerular filtration rate, indirectly suggesting that the Nanobody was mainly eliminated via the kidneys. When linked to an anti-albumin Nanobody, a 376-fold decrease in clearance was observed, resulting in a terminal half-life of 4.9 days, corresponding to the expected species albumin half-life. Similar conclusions were drawn for (non-) half-life extended mono-, bi- and trimeric Nanobodies in mice or rabbits, suggesting that these kinetic principles apply across species. Applying this knowledge to species translation and study design is crucial for successful pre-clinical development of novel therapeutic Nanobody candidates.
\end{abstract}

Keywords: nanobodies; pharmacokinetics; albumin; half-life extension; pre-clinical 


\section{Introduction}

Nanobodies are antigen-binding, single variable domain proteins derived from naturally-occurring heavy chain only antibodies [1]. As hydrophobic interaction with a light chain is not required, Nanobodies are highly soluble, physicochemically stable and can be produced efficiently with high yields in eukaryotic (including yeast and mammalian) or prokaryotic host organisms. The single domain nature and small size allow easy formatting by genetic fusion into multimeric constructs with multiple specificities [2,3]. Owing to these favorable properties, several Nanobodies are being developed for a wide variety of therapeutic applications $[4,5]$.

In monomeric format, Nanobodies $( \pm 15 \mathrm{kDa})$ are approximately ten times smaller than monoclonal antibodies $( \pm 150 \mathrm{kDa})$. Small proteins are expected to be filtered by the renal glomerulus. The fraction of compound that can pass through the glomerular filter depends on the size, charge, and shape of the macromolecule [6]. Filtration occurs freely for compounds smaller than approximately $10 \mathrm{kDa}$ [7]. The fractional clearance decreases with increasing size, and is expected to become marginal for molecules with hydrodynamic radii above 3.5 to $6 \mathrm{~nm}$ [6], as the effective pore size of the glomerulus is estimated around 8 to $10 \mathrm{~nm}$ in diameter [7]. Given a similar size, cationic compounds are more easily filtered than neutral or anionic compounds due to the strong net negative charge at the site of the glomeruli, as demonstrated by dextran (radius of $\pm 3.6 \mathrm{~nm}$ ) and horse radish peroxidase (radius of $\pm 3 \mathrm{~nm}$ ) ranging in pI below 4 up to 9.2 [6,8]. Additionally, the fractional clearance of linear flexible molecules such as polyvinylpyrrolidone and dextran appears to be higher than for proteins similar in size [6]. Often, albumin $(66.4 \mathrm{kDa}$ [9]) is referred to as a good estimate for the molecular weight cut-off of the glomerulus [7]. The sieving coefficient at which albumin is filtered through the glomerulus is small, estimated at $0.062 \%[10,11]$ to $0.19 \%$ in rats [12]. Taken together, Nanobodies are expected to be filtered, although not freely, by the glomerulus. Indeed, proteins of similar size, such as nartograstim (19 kDa) and filgrastim $(19 \mathrm{kDa})$ have been reported to be extensively cleared by renal excretion in rats [13,14].

Proteins larger than albumin are thought to be mainly eliminated through catabolism via the lysosomal or ubiquitin protein degradation pathways [7]. Both albumin and immunoglobulin $\mathrm{G}(\operatorname{IgG})$ are protected from lysosomal degradation by binding to the neonatal $\mathrm{Fc}$ receptor $(\mathrm{FcRn})$ which is highly expressed by many cells $[15,16]$. Binding to this receptor occurs strictly in a pH-dependent manner: following pinocytosis, IgG and albumin bind to FcRn in the acidic environment of the endosomes, but not at physiological $\mathrm{pH}$. By binding to FcRn in the acidified endosomes, intact IgG and albumin can be recycled back to the cell surface resulting in exocytosis and release from FcRn extracellularly at neutral $\mathrm{pH}$ [17]. As a result, these molecules show high residence times, with reported half-lives in human of approximately 17-19 days [18] for albumin and 21 days for IgG1, IgG2 and IgG4 [19]. Attempts have been made to extend the half-life of small proteins and peptides by limiting renal elimination and/or benefiting from the FcRn salvaging pathway, e.g., through (i) fusion of the protein to albumin [20] or to the Fc fragment of IgG [21], (ii) covalently binding to polyethylene glycol increasing its hydrodynamic radius and stability [22], or (iii) targeting endogenous albumin reversibly [17,23-25]. Similarly, anti-albumin Nanobodies have been linked to other Nanobody constructs into multi-specific formats [26]. However, only limited fragmentary information on the detailed pharmacokinetic (PK) behavior of Nanobodies (anti-albumin half-life extended or not) is currently available in literature [5]. An integrated understanding of factors affecting the disposition of Nanobodies of different formats will enable to implement more robust pre-clinical and clinical study designs. 
The aim of the present manuscript is to elaborate on the general pre-clinical serum PK of non-halflife and anti-albumin half-life extended Nanobodies. For this purpose, seven Nanobodies of varying format and size were administered intravenously (i.v.) in mice, rabbits or cynomolgus monkeys and the resulting serum PK profiles were analyzed and interpreted.

\section{Results}

Following a single i.v. bolus administration, the serum PK of seven Nanobodies composed of varying constructs (mono-, di-, or trimeric Nanobodies) of a combination of irrelevant ('Irr': no target binding) and albumin binding ('Hle': Half-life extension) building blocks, were evaluated in mice, rabbits or cynomolgus monkeys. An overview of the test items and the performed experiments is provided in Table 1. Mean ( $\mathrm{n}=3 ; \pm$ standard deviation) serum concentration PK profiles are shown in Figure 1.

Table 1. Overview of the main characteristics of the Nanobody test items and the performed animal studies.

\begin{tabular}{cccccccccc}
\hline $\begin{array}{c}\text { Experi } \\
\text {-ment }\end{array}$ & Nanobody & $\begin{array}{c}\text { MW } \\
\text { (kDa) }\end{array}$ & $\begin{array}{c}\text { Mono-di- } \\
\text { trimeric }\end{array}$ & pI & Dose (i.v.) & Species & $\begin{array}{c}\text { Average } \\
\text { body } \\
\text { weight (kg) }\end{array}$ & $\begin{array}{c}\text { Ko for } \\
\text { species } \\
\text { albumin }\end{array}$ & $\begin{array}{c}\text { Assay } \\
\text { LLOQ } \\
\text { (ng/mL) }\end{array}$ \\
\hline 1 & Hle1 & 16 & Mono- & 6.4 & $100 \mu \mathrm{g}$ & Mouse & 0.019 & $73 \mathrm{nM}$ & 52 \\
2 & Irr1 & 16 & Mono- & 5.5 & $100 \mu \mathrm{g}$ & Mouse & 0.019 & - & 5 \\
3 & Irr1-Hle1 & 31 & Di- & 6.1 & $100 \mu \mathrm{g}$ & Mouse & 0.019 & $470 \mathrm{nM}$ & 40 \\
4 & Irr1-Irr1 & 31 & Di- & 5.4 & $100 \mu \mathrm{g}$ & Mouse & 0.019 & - & 15 \\
5 & Irr2-Hle2-Irr2 & 41 & Tri- & 9.0 & $1.2 \mathrm{mg} / \mathrm{kg}$ & Mouse & 0.019 & $180 \mathrm{nM}$ & 40 \\
6 & Irr2-Hle2-Irr2 & 41 & Tri- & 9.0 & $2.5 \mathrm{mg} / \mathrm{kg}$ & Rabbit & 1.99 & No binding & 40 \\
7 & Irr3-Hle2 & 26 & Di- & 7.1 & $2 \mathrm{mg} / \mathrm{kg}$ & Monkey & 2.37 & ND $^{\mathrm{a}}$ & 1.2 \\
8 & Irr3 & 13 & Mono- & 5.8 & $2 \mathrm{mg} / \mathrm{kg}$ & Monkey & 2.40 & - & 8 \\
\hline
\end{tabular}

MW: Molecular Weight; Mono-di-trimeric: refers to the number of building blocks comprising one full Nanobody; pI: Isoelectric point; $\mathrm{K}_{\mathrm{D}}$ : Dissociation Constant; LLOQ: Lower Limit of Quantification of PK assay; Hle: half-life extended (anti-albumin) building block; Irr: irrelevant (no target binding) building block. a: ND, Not Determined: the $\mathrm{K}_{\mathrm{D}}$ based on human serum albumin was $31 \mathrm{nM}$ and is expected to be similar to cynomolgus monkey serum albumin.

The half-life extended Nanobodies Hle1, Irr1-Hle1 and Irr2-Hle2-Irr2 (Table 1, mono-, di- and trimeric, respectively) in mice and Irr3-Hle2 (dimeric) in monkeys showed a clear prolonged exposure compared with the non-half-life extended Nanobodies Irr1 and Irr1-Irr1 (mono- and dimeric) in mice, Irr2-Hle2-Irr2 (trimeric) in rabbits and Irr3 (monomeric) in monkeys: for half-life extended Nanobodies, serum levels were detectable up to 10 days in mice and 35 days in monkeys (Figure 1, Panel A), while for non-half-life extended Nanobodies, levels were below the quantification limit within the first day following administration (Figure 1B). For all test items, the ELISA lower quantification limits ranged from 5 to $52 \mathrm{ng} / \mathrm{mL}$ (Table 1). The impact of half-life extension seemed comparable for monomeric versus dimeric Nanobodies following equal dosing (Figure 1C,D, respectively) with serum levels detectable up to 4 days after i.v. administration of $100 \mu \mathrm{g}$. Similarly, in monkeys following $2 \mathrm{mg} / \mathrm{kg}$ i.v. dosing, serum levels of Nanobody Irr3 were only quantifiable up to $8 \mathrm{~h}$, while the addition of an albumin binding block prolonged the exposure to 35 days (Figure 1F). Nanobody Irr2-Hle2-Irr2 displayed a prolonged exposure in mice versus rabbits (Figure 1F) even though the dose for the rabbits was more than double than for the mice $(2.5 \mathrm{mg} / \mathrm{kg} v s .1 .2 \mathrm{mg} / \mathrm{kg})$. The dissociation constant $\left(\mathrm{K}_{\mathrm{D}}\right)$ of Irr2-Hle2Irr2 for mouse serum albumin was $180 \mathrm{nM}$, while Irr2-Hle2-Irr2 could not bind to rabbit serum albumin 
(Table 1). No signs of emerging PK-altering anti-drug antibodies were apparent, based on the linearity of the terminal phase of the PK profiles.

As all Nanobodies displayed a biphasic exponential decline in serum concentration levels, a bicompartmental model was used to fit the data and the resulting PK parameters are shown for each experiment in Table 2 .
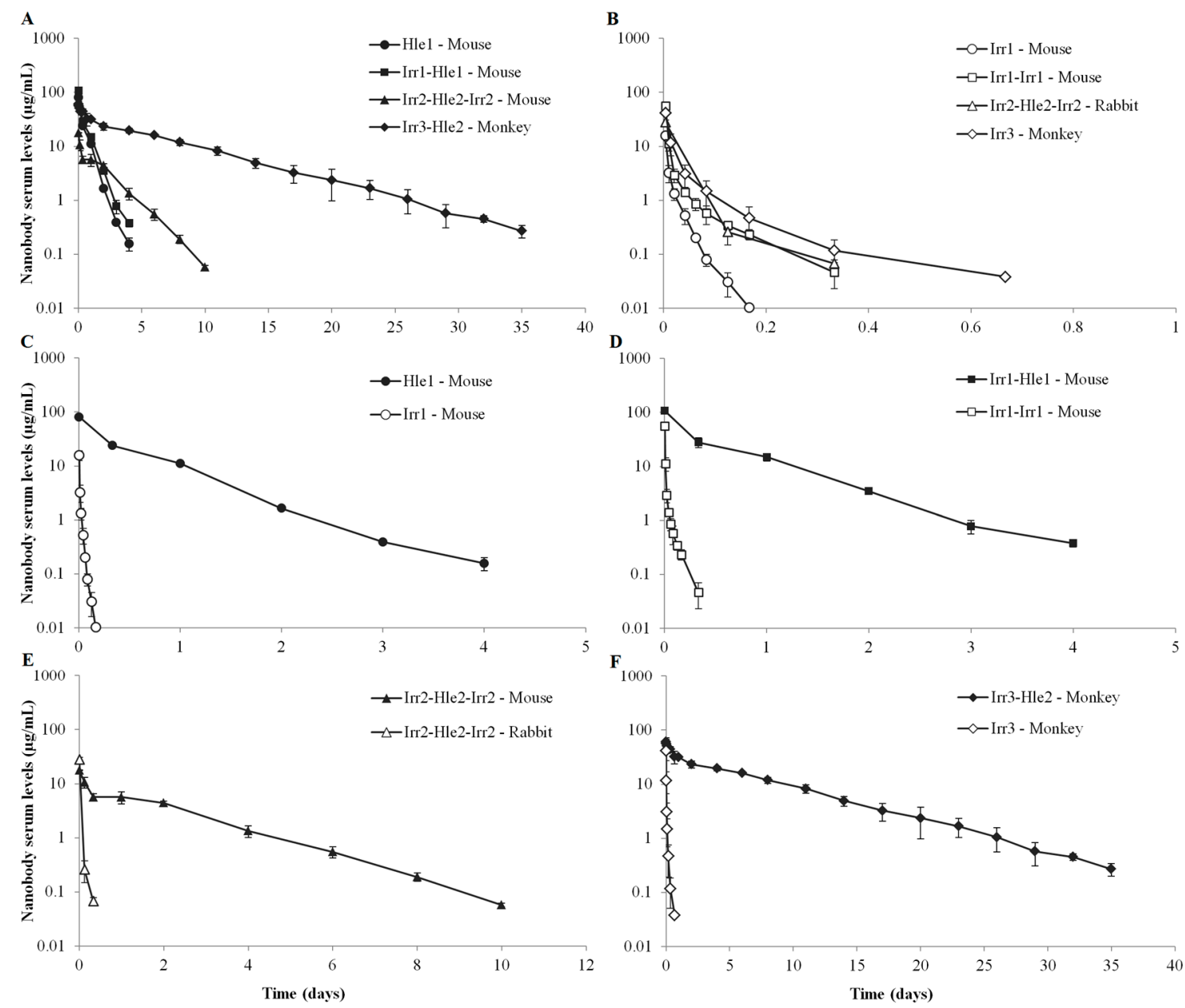

Figure 1. Mean observed ( $\mathrm{n}=3^{*} ; \pm$ standard deviation) serum concentrations $(\mu \mathrm{g} / \mathrm{mL})$ in function of time (days) of seven Nanobodies tested in mice, rabbits or cynomolgus monkeys after single intravenous (i.v.) administration (semi-log scale). Panels $(\mathbf{A}, \mathbf{B})$ versus $(\mathbf{C}-\mathbf{F})$ represent the same dataset, plotted differently for interpretation purposes. For clarity, antialbumin half-life extended Nanobodies ((A); Filled symbols) are plotted on a separate timescale than non-half-life extended Nanobodies ((B); Open symbols). The impact of half-life extension on monomeric (C) and dimeric (D) Nanobodies (Hle1 vs. Irr1 and Irr1-Hle1 vs. Irr1-Irr1, respectively) tested at a fixed dose of $100 \mu \mathrm{g}$ in mice is shown. The necessity for high affinity of an anti-albumin Nanobody (Irr2-Hle2-Irr2) for the respective species albumin is shown for mice (Table 1, dissociation constant $\mathrm{K}_{\mathrm{D}}=180 \mathrm{nM} ; 1.2 \mathrm{mg} / \mathrm{kg}$ dosing) versus rabbits (no albumin binding; $2.5 \mathrm{mg} / \mathrm{kg}$ dosing) in (E). The impact of half-life extension by linking an anti-albumin Nanobody (Hle2) to an irrelevant Nanobody (Irr3) tested in cynomolgus monkeys $(2 \mathrm{mg} / \mathrm{kg})$ is shown $(\mathbf{F})$. * Except for $\operatorname{Irr} 3$ after 16 hours $(\mathrm{n}=1)$ and Irr3-Hle2 after 32 and 35 days $(n=2)$ post administration, due to values below the limit of quantification. 
Table 2. Main serum PK parameters of tested Nanobodies in mice, rabbits or cynomolgus monkeys after single i.v. administration.

\begin{tabular}{|c|c|c|c|c|c|c|c|c|c|c|c|c|c|c|c|c|c|}
\hline \multirow[b]{2}{*}{ Parameter } & \multirow[b]{2}{*}{ Units $^{\text {a }}$} & \multicolumn{2}{|c|}{$\begin{array}{c}\text { Hle1 } \\
\text { Mouse }\end{array}$} & \multicolumn{2}{|c|}{$\begin{array}{c}\text { Irr1 } \\
\text { Mouse }\end{array}$} & \multicolumn{2}{|c|}{$\begin{array}{c}\text { Irr1-Hle1 } \\
\text { Mouse }\end{array}$} & \multicolumn{2}{|c|}{$\begin{array}{c}\text { Irr1-Irr1 } \\
\text { Mouse }\end{array}$} & \multicolumn{2}{|c|}{$\begin{array}{c}\text { Irr2-Hle2-Irr2 } \\
\text { Mouse }\end{array}$} & \multicolumn{2}{|c|}{$\begin{array}{c}\text { Irr2-Hle2-Irr2 } \\
\text { Rabbit }^{\text {b }}\end{array}$} & \multicolumn{2}{|c|}{$\begin{array}{l}\text { Irr3-Hle2 } \\
\text { Monkey }^{c}\end{array}$} & \multicolumn{2}{|c|}{$\begin{array}{c}\text { Irr3 } \\
\text { Monkey }^{c}\end{array}$} \\
\hline & & Estimate & RSE\% & Estimate & RSE\% & Estimate & RSE\% & Estimate & RSE $\%$ & Estimate & RSE\% & Estimate & RSE\% & Mean & $\% \mathrm{CV}$ & Mean & $\% \mathrm{CV}$ \\
\hline $\mathrm{Vc}$ & $\mathrm{mL} / \mathrm{kg}$ & 64.0 & 36 & 171 & 40 & 47.0 & 59 & 49.1 & 27 & 64.7 & 21 & - & - & 34.4 & 11 & 41.4 & 38 \\
\hline $\mathrm{Vp}$ & $\mathrm{mL} / \mathrm{kg}$ & 48.3 & 172 & 192 & 32 & 54.9 & 413 & 152 & 27 & 54.5 & 32 & - & - & 17.9 & 43 & 92.0 & 19 \\
\hline \multirow[t]{2}{*}{$\mathrm{CL}$} & $\mathrm{mL} /\left(\mathrm{day}^{*} \mathrm{~kg}\right)$ & 176 & 36 & 29425 & 18 & 135 & 103 & 8102 & 14 & 60.4 & 8 & 2907 & - & 7.61 & 8 & 2857 & 42 \\
\hline & $\mathrm{mL} /(\min * \mathrm{~kg})^{\mathrm{d}}$ & 0.122 & 36 & 20.4 & 18 & 0.094 & 103 & 5.63 & 14 & 0.042 & 8 & 2.02 & - & 0.005 & 8 & 1.98 & 42 \\
\hline \multirow[t]{2}{*}{ Q } & $\mathrm{mL} /\left(\right.$ day*kg $\left.^{*}\right)$ & 312 & 553 & 8028 & 41 & 427 & 1561 & 2215 & 31 & 493 & 107 & - & - & 50 & 46 & 1331 & 37 \\
\hline & $\mathrm{mL} /(\min * \mathrm{~kg})^{\mathrm{d}}$ & 0.217 & 553 & 5.58 & 41 & 0.296 & 1561 & 1.54 & 31 & 0.342 & 107 & - & - & 0.034 & 46 & 0.924 & 37 \\
\hline AUC & day* $\mu \mathrm{g} / \mathrm{mL}$ & 29.8 & 36 & 0.179 & 18 & 38.9 & 103 & 0.650 & 14 & 19.9 & 8 & 0.860 & - & 264 & 8 & 0.793 & 43 \\
\hline $\mathrm{t}_{1 / 2 \text {, beta }}$ & day & 0.494 & 11 & 0.022 & 8 & 0.575 & 10 & 0.062 & 8 & 1.40 & 5 & 0.107 & - & 4.89 & 15 & 0.080 & 29 \\
\hline $\mathrm{V}_{\mathrm{ss}}$ & $\mathrm{mL} / \mathrm{kg}$ & 112 & 69 & 363 & 33 & 102 & 199 & 201 & 26 & 119 & 10 & 107 & - & 52.3 & 9 & 133 & 25 \\
\hline
\end{tabular}

Vc: Central volume of distribution; Vp: peripheral volume; CL: Clearance; Q: Inter-compartmental flow; AUC: Area under the curve; $\mathrm{t}_{1 / 2}$,beta: terminal or beta phase half-life; $\mathrm{V}_{\mathrm{ss}}$ : Volume of distribution at steady state. \%RSE: Relative Standard Error, calculated by $100 *$ standard error / parameter estimate; \%CV: Coefficient of Variation, calculated by $100 *$ standard deviation / mean value. ${ }^{a}$ Units were adjusted for body weight assuming a $19 \mathrm{~g}$ mouse for the mice studies with fixed dosing of $100 \mu \mathrm{g}$ (Hle1; Irr1; Irr1-Hle1; Irr1-Irr1). ${ }^{\mathrm{b}}$ Due to limited data, parameters were estimated via non-compartmental analysis using two data-points only for the terminal phase (Parameter uncertainty expected to be high). ${ }^{\mathrm{c}}$ In contrast with mouse and rabbit (mean profiles), PK profiles in monkeys were analyzed individually ( $\mathrm{n}=3$ per treatment) and mean (\%CV) of individual parameters are shown. ${ }^{\mathrm{d}}$ For clarity in interpreting CL and Q, these parameters are expressed both in $\mathrm{mL} /($ day* $\mathrm{kg})$ and in $\mathrm{mL} /(\mathrm{min} * \mathrm{~kg})$. 
As the obtained PK profile in rabbits consisted of data at only three time-points, two-compartmental PK analysis could not be performed. These data were analyzed via non-compartmental analysis, with time-points at $3 \mathrm{~h}$ and $8 \mathrm{~h}$ post-administration selected as terminal phase. As a result, the obtained parameters for Irr2-Hle2-Irr2 in rabbits are not expected to be estimated accurately and should therefore be interpreted with caution (Table 2). In mice, PK parameters based on mean profiles were estimated with acceptable precision, with relative standard errors (RSE) generally remaining below $40 \%$, except for inter-compartmental flow (Q) and for most parameters of Nanobody Irr1-Hle1 (Table 2). In contrast to mice and rabbits (mean profiles), complete individual PK profiles were obtained and analyzed individually in monkeys. The PK parameters were estimated with acceptable precision (RSE below 40\%) in the individual monkeys, except for Q (RSE up to 190\%) in most animals and for peripheral volume $(\mathrm{Vp})$ in one animal (60\% RSE). The resulting mean PK parameters displayed relatively small variation coefficients (CV), below 50\% for all estimated parameters (Table 2), indicating that the between animal variability ( $n=3$, per treatment) was limited. Overall, these precision results showed that the main PK parameters, central distribution volume $(\mathrm{Vc})$ and clearance $(\mathrm{CL})$, were well estimated. Distribution parameters $\mathrm{Vp}$ and $\mathrm{Q}$ were estimated less precisely, especially for half-life extended Nanobodies, suggesting that perhaps a more extensive sampling in the initial phase of the PK profile would be required when improved parameter estimation of $\mathrm{Vp}$ and $\mathrm{Q}$ is of particular interest.

The distribution volume at steady state $\left(\mathrm{V}_{\mathrm{ss}}\right)$ of the half-life extended Nanobodies was low, ranging from $52.3 \mathrm{~mL} / \mathrm{kg}$ in monkeys to $119 \mathrm{~mL} / \mathrm{kg}$ in mice (Table 2), suggesting that these Nanobodies are mainly confined to the vascular space. In contrast, non-half-life extended Nanobodies showed a two to three-fold increase in $\mathrm{V}_{\text {ss }}$, up to $363 \mathrm{~mL} / \mathrm{kg}$ for the monomeric Nanobody Irr1, suggesting increased tissue distribution. However, non-half-life extended Nanobodies showed a dramatic increase in clearance, compared with their anti-albumin counterparts, with the highest clearance, $20.4 \mathrm{~mL} /(\mathrm{min} * \mathrm{~kg})$, observed for Irr1 which constitutes a 167-fold clearance increase compared with the monomeric antialbumin Nanobody Hle1. In cynomolgus monkeys, a 376-fold increase in clearance was observed for Irr3 compared with Irr3-Hle2. As a consequence, estimated exposure (AUC) and half-life of the terminal or beta phase ( $\left.\mathrm{t}_{1 / 2, \text { beta }}\right)$ was markedly lower for non-half-life extended Nanobodies (Table 2$)$. Similarly, the estimated clearance drastically increased (48-fold), with corresponding decrease in AUC (48-fold decrease in dose normalized AUC), when Irr2-Hle2-Irr2 was administered to rabbits (i.v. $2.5 \mathrm{mg} / \mathrm{kg}$ ) compared with mice (i.v. $1.2 \mathrm{mg} / \mathrm{kg}$ ), as Hle2 binds strongly to mouse albumin but not to rabbit albumin.

Overall, these results clearly indicate that half-life extension via anti-albumin binding proved successful in decreasing the total Nanobody clearance resulting in prolonged exposure in terms of estimated AUC and measurable serum levels in mice and monkeys, as shown by a set of different Nanobody constructs.

Regarding safety, no signs of local or systemic intolerance were observed during the in vivo phase of the studies, and none of the animals died prematurely.

\section{Discussion}

In order to demonstrate the general PK behaviour of different Nanobody formats, serum levels were monitored for seven Nanobodies, composed of anti-albumin binding or irrelevant (no target binding) building blocks, administered intravenously in different animal species. For half-life extension purposes, albumin 
targeting is desired due to its abundance (ranging between 35 to $50 \mathrm{~g} / \mathrm{L}$ in normal human plasma [9]) and its long terminal elimination half-life in humans, estimated at approximately 17-19 days [18,27].

\subsection{Serum Pharmacokinetics of Non-Half-Life Extended Nanobodies}

The total clearance observed for the non-half-life extended Nanobodies was faster than for albumin binding formats. Due to their small size, unbound Nanobodies are rapidly cleared from the systemic circulation through the kidneys by glomerular filtration, as demonstrated by the high percentage of radioactivity recovered in the kidneys shortly after dosing and the rapid decline in total radioactivity recovery [26,28-30]. Filtration occurs freely for compounds smaller than approximately $10 \mathrm{kDa}$ [7], while for larger proteins, the filtration rate decreases upon increasing size and decreasing isoelectric point (pI) [6,8]. Human serum albumin $(66.4 \mathrm{kDa}$; $\mathrm{pI}=5)$ is often referred to as a good estimate of molecular weight cut-off of the glomerulus [6,7]. Since the non-half-life extended Nanobodies tested in our experiments were smaller than albumin (ranging from 13 to $41 \mathrm{kDa}$ ) and had a higher pI (ranging from 5.4 to 9), it is expected that a significant fraction of Nanobody could pass the glomerular filter at a high rate. As with most physiological rates and blood flows, the glomerular filtration rate (GFR) increases with decreasing body weight across species, from an estimated $14 \mathrm{~mL} /(\mathrm{min} * \mathrm{~kg})$ in a $20 \mathrm{~g}$ mouse up to $1.8 \mathrm{~mL} /\left(\mathrm{min}^{*} \mathrm{~kg}\right)$ for a $70 \mathrm{~kg}$ human [31]. Interestingly, the estimated total clearance of 2.0 and $20.4 \mathrm{~mL} /\left(\mathrm{min}^{*} \mathrm{~kg}\right)$ of monomeric Nanobodies Irr3 (13 kDa; evaluated in cynomolgus monkeys) and $\operatorname{Irr} 1$ (16 kDa; evaluated in mice) approximated the GFR in the corresponding species, 2.1 and $14 \mathrm{~mL} /\left(\mathrm{min}^{*} \mathrm{~kg}\right)$ for monkeys and mice, respectively [31]. Once filtered, Nanobody removal is expected to occur via urine, either degraded or intact, or through catabolism in proximal tubule cells.

Of note, serum PK can only indirectly suggest the dominance of renal filtration, and total drug clearance remains a combination of both renal elimination and catabolism [7]. Moreover, due to the rapid Nanobody elimination, it becomes particularly challenging to accurately estimate clearance, as the dominant fraction of the area under the curve (AUC) is expected to occur during the initial phase of the PK profile, and immediately upon administration. To illustrate this, the PK profile of Irr1 in mice was analyzed via non-compartmental PK analysis (NCA), which revealed that the fraction of the total AUC originating from back-extrapolation from the first time-point ( 5 min post-administration) to time of administration was large: $43 \%$ of the total AUC. As this back-extrapolation resulted in a lower estimation of concentration at time $0\left(\mathrm{C}_{0}\right)$ than expected, it is likely that the PK analysis underestimates the AUC and thus overestimates the true clearance. For example, upon i.v. administration, the $\mathrm{C}_{0}$ of a Nanobody is expected to be approximately similar to the administered dose divided by the plasma volume, which corresponds to $100 \mu \mathrm{g} / \mathrm{mL}$ for a fixed dose of $100 \mu \mathrm{g}$ in mice assuming a plasma volume of $1 \mathrm{~mL}$ [31]. Indeed, the $\mathrm{C}_{0}$ for half-life extended Nanobodies Hle1 and Irr1-Hle1, was estimated via NCA at 82 to $109 \mu \mathrm{g} / \mathrm{mL}$, respectively, following a fixed dose of $100 \mu \mathrm{g}$ in mice. Including a fictive data-point in the PK profile of Irr1, $C_{0}$ fixed at $100 \mu \mathrm{g} / \mathrm{mL}$, resulted in a decrease in estimated clearance from 19.1 to $13.7 \mathrm{~mL} /(\mathrm{min} * \mathrm{~kg})$ via NCA, which closely approximates the GFR estimated at $14 \mathrm{~mL} /\left(\mathrm{min}^{*} \mathrm{~kg}\right)$ in mice [31]. Furthermore, this sensitivity analysis illustrates that the choice of PK sampling schedule can have a large potential impact on the estimated parameters for non-half-life extended Nanobodies.

As mentioned, the renal contribution to total CL increases with decreasing molecular weight $[6,7,32]$. Thus, as expected for molecules of similar pI (5.4-5.5), the clearance of the dimeric Nanobody Irr1-Irr1 
$(31 \mathrm{kDa})$ was smaller than that of the monomeric Nanobody Irr1 $(16 \mathrm{kDa})$, estimated at 5.6 and $20.4 \mathrm{~mL} /(\mathrm{min} * \mathrm{~kg})$, respectively. Similarly, the trimeric Nanobody Irr2-Hle2-Irr2 (41 kDa; pI = 9) tested in rabbit and with building block Hle2 unable to bind to rabbit albumin, showed a fast clearance $(2.0 \mathrm{~mL} /(\mathrm{min} * \mathrm{~kg}))$ as expected, but slower than the reported GFR in rabbits $(3.1 \mathrm{~mL} /(\mathrm{min} * \mathrm{~kg})[31])$.

In this study, irrelevant Nanobodies were selected to demonstrate the general PK properties of Nanobodies. However, it should be considered in a case-by-case manner that target binding can alter the Nanobody pharmacokinetics. For example, data on the von Willebrand factor (VWF) targeting Nanobody ALX-0081 in mice showed that, in contrast to unbound Nanobody where the percentage of radioactivity was elevated in the kidneys, radioactivity of VWF-bound Nanobody accumulated mainly in the liver [28], similar to free VWF evaluated in VWF-deficient mice [33]. These data suggest that the disposition of ALX-0081 is altered by binding to its target.

\subsection{Serum Pharmacokinetics of Half-Life Extended Nanobodies}

The anti-albumin half-life extended Nanobodies showed a substantial decrease in total clearance compared to their non-half-life extended counterparts: a 167-fold, 60-fold and 376-fold decrease in clearance was observed for Hle1 versus Irr1 in mice, Irr1-Hle1 versus Irr1-Irr1 in mice and Irr3 versus Irr3-Hle2 in cynomolgus monkeys, respectively. Although the latter comparison in monkeys evaluates the same monomeric Nanobody (Irr3) with or without the anti-albumin moiety (Hle2) resulting in a difference in molecular weight (13 kDa for Irr3 and $26 \mathrm{kDa}$ for Irr3-Hle2), this comparison is still considered to be valid as the PK of the anti-albumin Nanobodies is expected to be mainly driven by albumin binding rather than by the molecular weight of the Nanobody, as demonstrated by the similar PK of Nanobodies Hle1 (16 kDa) and Irr1-Hle1 (31 kDa) in mice (Figure 1A; Table 2). Presumably, due to the size of the Nanobody-albumin complex, the fraction of complex that is cleared by renal excretion would be limited. Moreover, as demonstrated for albumin, filtered complex might be salvaged back into circulation via tubular re-absorption mediated by albumin neonatal $\mathrm{Fc}$ receptor ( $\mathrm{FcRn}$ ) recycling [15,34]. The protection of albumin through FcRn- endothelial cells $[15,35,36]$. The half-life of albumin between species was found to approximately recycling is not restricted to the kidneys, as FcRn is highly expressed on many cell types throughout the body, including vascular adhere to the following allometric scaling relationship: albumin half-life (days) $=3.75 *$ Body Weight $(\mathrm{kg})^{0.368}$ [37]. Applying this relationship to a $19 \mathrm{~g}$ mouse and a $2.4 \mathrm{~kg}$ monkey, the observed average body weight in our animal studies, suggests an albumin half-life of 0.87 and 5.2 days for these mice and monkeys, respectively. These values closely matched with the observed average terminal half-life for the three tested half-life extended Nanobodies in mice (Hle1, Irr1-Hle1 and Irr2-Hle2-Irr2) of 0.82 days, and the terminal half-life of Irr3-Hle2 in cynomolgus monkeys of 4.9 days. These data suggest that the half-life of anti-albumin Nanobodies was successfully increased by assuming the PK behavior of albumin in the respective species through albumin binding and subsequent protection by FcRn-recycling. At $\mathrm{K}_{\mathrm{D}}$ values for murine albumin between $73 \mathrm{nM}$ (Hle1) and $470 \mathrm{nM}$ (Irr1-Hle1), the PK profiles were highly similar (Figure 1, Panels A, $\mathrm{C}$ and D), suggesting that affinities below $470 \mathrm{nM}$ do not further improve the Nanobody kinetics in mice. Although the clearance was slightly higher for Hle1 versus Irr1-Hle1, no conclusion can be drawn from this observation given the small difference in clearance (176 and $135 \mathrm{~mL} /($ day*kg), respectively), the estimated precision of clearance (36 and 103\% RSE, respectively) and the inherent expected inter-subject 
variability of PK parameters. The allometric albumin half-life relationship [37] predicts an albumin half-life of 4.8 days for a $2 \mathrm{~kg}$ rabbit. However, the estimated terminal half-life of Irr2-Hle2-Irr2 in rabbits was only 0.13 days. These results were as expected since there is no cross-reactivity of this Nanobody to rabbit albumin, demonstrating the importance of assessing not only the affinity of an anti-albumin therapeutic Nanobody for its therapeutic target but also for albumin in each animal species of interest in a pre-clinical development setting.

Due to the rapid clearance of non-half-life extended Nanobodies, clearance was estimated to be larger than inter-compartmental flow, while the opposite was observed for half-life extended Nanobodies. Although for both types, the PK profiles were characterized by a bi-phasic decline, this observation suggests that in contrast to anti-albumin Nanobodies where, at distribution equilibrium, the body is expected to behave as a single compartment, the kinetics of non-half-life extended Nanobodies would be distribution-limited with the initial phase of the PK profile mainly corresponding to the fast elimination and the terminal phase driven mainly by distribution. Therefore, in the specific case for non-half-life extended Nanobodies, the terminal half-life is not expected to reflect the elimination half-life. This further emphasizes the dominance of the first hour(s) after administration for an accurate estimation of exposure and clearance for non-half-life extended Nanobodies. The estimated volume of distribution at steady state was small for the half-life extended Nanobodies ranging from $52 \mathrm{~mL} / \mathrm{kg}$ in monkeys to $119 \mathrm{~mL} / \mathrm{kg}$ in mice, suggesting that Nanobody-albumin complex was mostly restricted to the central compartment with limited tissue distribution, similarly to monoclonal antibodies [38]. Non-half-life extended Nanobodies showed a two to three-fold increase in steady state volume of distribution, suggesting an increase, albeit limited, in distribution due to the smaller size of these unbound Nanobodies. Potentially, tissue distribution could increase further for Nanobodies binding to a target present outside the vascular space, due to high affinity target binding and retention of drug at the target tissue sites [38].

Both albumin as well as IgG benefit from FcRn recycling resulting in an increase in residence time, albeit with different binding sites and stoichiometry [15,17]. However, in contrast to species with higher body weight, the half-life of albumin in rodents of approximately 1 to 2.5 days is typically lower than that observed for many IgG's, ranging from several days to weeks [15,35,37,39-42]. For example, Deng et al. [39] reported an observed clearance of 3-16 mL/(day* $\mathrm{kg})$ in mice and 5-12 $\mathrm{mL} /($ day* $\mathrm{kg})$ in cynomolgus monkeys for 13 monoclonal antibodies. These values are markedly lower than the clearance observed for our Nanobodies in mice $(60.4-176 \mathrm{~mL} /($ day* $\mathrm{kg})$ ), thus leading to higher expected terminal half-lives given similar distribution. In contrast, in monkeys, the clearance of Nanobody Irr3-Hle2 $(7.61 \mathrm{~mL} /($ day* $\mathrm{kg})$ ) was similar to the reported clearance of conventional antibodies. Additionally, monoclonal antibody half-life can be very variable depending on the extent of sequence optimization (humanization), evaluated species, isotype or affinity for FcRn. For example, human IgG1 was found to bind more strongly to murine FcRn than mouse IgG1, while binding of mouse IgG1 to human FcRn was limited [43]. In light of in vivo proof-of-concept studies in pre-clinical animal models, it is imperative that these potential differences are taken into account. It is therefore recommended that the PK behavior of both antibody and anti-albumin Nanobody are well characterized in the test species allowing optimal dosing to reach the desired exposure in efficacy studies and allowing more reliable cross-species exposure predictions. 


\subsection{Conclusion}

In conclusion, the serum pharmacokinetics of non-half-life extended Nanobodies are dominated by a high total clearance, approximating the species glomerular filtration rate for monomeric constructs, suggesting extensive renal elimination. In contrast, the clearance of anti-albumin Nanobodies is much lower, owing to (i) the larger size of the Nanobody-albumin complex reducing glomerular filtration and (ii) the presumed protection of the complex by albumin salvaging via the neonatal Fc receptor. As such, these half-life extended Nanobodies appear to adopt the kinetics of albumin as demonstrated by the strong similarity between observed Nanobody half-life and predicted albumin half-life in different species. In addition to clearance, the distribution characteristics of Nanobodies can also be modulated, as a two to three-fold increase in steady state volume of distribution was observed for non-half-life extended versus anti-albumin Nanobodies. One major asset of the Nanobody technology is the flexibility to choose a format that reflects the specific kinetic needs: for chronic disease indications, where prolonged exposure is crucial, a half-life extended construct can be generated, while in acute indications non-half-life extended formats might show preferential kinetics. This advantage is demonstrated by the multiple Nanobodies including examples of both formats that are currently in clinical development in a wide array of therapeutic areas, including inflammation, infectious diseases, and hematology [3,44-46].

\section{Experimental Section}

\subsection{Test Item Nanobodies}

Seven Nanobodies, composed of a combination of irrelevant (no target binding) and half-life extension (albumin binding) building blocks were generated as described by Arbabi Ghahroudi et al. [47]. Briefly, llamas were vaccinated with a target protein and RNA was extracted from peripheral blood lymphocytes. Genes for the variable domain of the heavy-chain only antibodies (VHH) were amplified from a cDNA library. Target binding Nanobody candidates were selected by panning phage libraries on plates coated with the target of interest. For the generation of multimeric Nanobodies, monomeric building blocks were genetically fused using flexible glycine-serine (GS) linkers. Nanobodies were produced in an Escherichia coli strain (Table 1, Experiments (Exp.) 1, 2, 7 and 8) or in a Pichia pastoris strain (Exp. 3, 4, 5 and 6). All seven Nanobodies intended for in vivo experiments were purified to endotoxin levels below $0.5 \mathrm{EU} / \mathrm{mL}$. The molecular weight of the final constructs ranged between $13 \mathrm{kDa}$ to $41 \mathrm{kDa}$. Anti-albumin building blocks were selected based on their capability to bind strongly to cynomolgus monkey and mouse albumin, while lack of binding was seen for rabbit albumin. Irrelevant Nanobody building blocks were selected based on either the absence of target (Irr1) or expected marginal target expression (Irr3), or the lack of target binding in the tested species (Irr2), to ensure that the experiments were not confounded by target-specific Nanobody disposition. In addition, the final selection of Nanobody building blocks and formatted Nanobodies was driven by (i) manufacturability (including yield, solubility and quality assessments), (ii) the impact of formatting on binding affinity and (iii) coverage of Nanobodies with a broad range in pI. An overview of the tested Nanobodies is provided in Table 1. 


\subsection{Animal Studies}

A set of in vivo experiments were set up to evaluate the PK of (non-) half-life extended Nanobodies in healthy female BALB/c mice, healthy female New-Zealand White rabbits or healthy male cynomolgus monkeys (Macaca fascicularis). The in vivo phase of experiments 5 and 6 (Table 1, Exp. 5 and 6) was executed by Charles River Laboratories (UK and France), while the other experiments were performed by LPT, Laboratory of Pharmacology and Toxicology (Germany).

The animal protocols prepared and followed by LPT were evaluated and approved by LPT's Animal Welfare Committee and the responsible local government veterinary office. They were in accordance with the German animal welfare law "Tierschutzgesetz" (TierSchG) and the EU "Directive on the protection of animals used for scientific purposes" valid at the time. Regarding Charles River Laboratories (UK), the animal protocols used were evaluated and approved by the Animal Welfare and Ethics Committee of Charles River Laboratories and were conducted under UK Home Office License No. PPL 60/3515, Drug Metabolism and Pharmacokinetics, Protocol Reference No. 1. The license was issued under the Animals (Scientific Procedures) Act 1986. Regarding Charles River Laboratories (France), the animal studies were evaluated and approved by the Charles River France Ethics Committee, and were conducted in accordance with the European Directive 86/609 (24 November 1986) and the French Decree (29 May 2001) and with the European and French Guidelines on animal welfare.

The animals were fed according to facility standards and drinking water was available ad libitum. The average body weight of the animals at time of dosing in the different experiments is provided in Table 1.

Sterile Nanobody solution prepared in Dulbecco's Phosphate Buffered Saline (D-PBS, GIBCO®, Thermo Fisher Scientific, UK) was administered by i.v. bolus injection via the tail vein (mice), ear artery (rabbits) and cephalic vein (monkeys) at a concentration of $0.5 \mathrm{mg} / \mathrm{mL}$ for mice and rabbits, and $2 \mathrm{mg} / \mathrm{mL}$ for cynomolgus monkeys. Whole blood samples $(\mathrm{n}=3$, per time-point, per experiment) were collected by cardiac vein or retrobulbar puncture for mice, puncture of the auricular artery for rabbits and of the cephalic or great saphenous vein for cynomolgus monkeys at the following sampling times: Pre-dose, $5 \mathrm{~min}, 8 \mathrm{~h}, 1$, 2, 3, 4, 7 and 11 days post-dose (Exp. 1 and 3); 5, 15, $30 \mathrm{~min}, 1,1.5,2,3,4$ and $8 \mathrm{~h}$ post-dose (Exp. 2 and 4); Pre-dose, $5 \mathrm{~min}, 3$ and 8 h, 1, 2, 4, 6, 8, 10, 12, 14 and 16 days post-dose (Exp. 5 and 6); Pre-dose, 5 and 20 min, 1, 2, 4, 8 and 16 h, 1, 2, 4, 6, 8, 11, 14, 17, 20, 23, 26, 29, 32, 35, 38, 41, 44, 47, 50, 53 and 56 days post-dose (Exp. 7 and 8).

Serum samples were prepared in fresh tubes by allowing the whole blood samples to clot for $0.5 \mathrm{~h}$ at room temperature (RT) (Exp. 1 to 4), between 1 to $3 \mathrm{~h}$ at RT (Exp. 5 and 6) or for $1 \mathrm{~h}$ at $37^{\circ} \mathrm{C}$ (Exp. 7 and 8), followed by centrifugation of the samples at $4^{\circ} \mathrm{C}$ for $3^{\prime}$ at 20,000 g (Exp. 1 to 4), 10' at 1,000 g (Exp. 5 and 6) or 10' at 10,000 g (Exp. 7 and 8). After separation of the upper phase, serum samples were stored at $-80^{\circ} \mathrm{C}$ prior to shipment and bioanalysis.

\subsection{Determination of Nanobody Serum Concentrations}

Total systemic Nanobody concentrations were determined with enzyme linked immunosorbent assays (ELISA). The test items were captured on a 96 well microtiter plate either with an IgG or a Nanobody directed against the test Nanobody (Table 1, Exp. 1 to 6) or with the human target protein the test item 
was generated against (Exp. 7 and 8). Detection was performed with either a horse radish peroxidase (HRP) labeled IgG (Exp. 1 to 4) or a biotinylated Nanobody followed by Streptavidin-HRP incubation (Exp. 5 and 6) or with an anti-Nanobody Rabbit polyclonal, followed by an HRP-Goat-Anti-Rabbit IgG incubation step (Exp. 7 and 8). The colorimetric change resulting from the catalysis of the chemical reaction with the added substrate 3,3',5,5'-Tetramethylbenzidine (TMB) was measured by a spectrophotometer at $450 \mathrm{~nm}$, after stopping the reaction with $1 \mathrm{M}$ hydrochloric acid. The Lower Limit of Quantification (LLOQ) for each assay is provided in Table 1.

\subsection{Pharmacokinetic Data Analysis}

Individual (cynomolgus monkey) or mean (mouse) serum concentration-time profiles after i.v. administration of the test items in each experiment were analyzed via two-compartmental (pre-programmed model 7) pharmacokinetic analysis using Phoenix WinNonlin version 6.3 (Pharsight, Certara, Princeton NJ, USA). Profiles were fitted using iterative re-weighting $\left(1 / \mathrm{y}^{2}\right)$, with $\mathrm{y}$ as the predicted serum concentration. The following PK parameters were estimated: primary parameters clearance (CL), central volume of distribution $(\mathrm{Vc})$, peripheral volume $(\mathrm{Vp})$ and inter-compartmental flow $(\mathrm{Q})$, and secondary parameters area under the curve (AUC), terminal or beta phase half-life ( $\mathrm{t}_{1 / 2, \text { beta }}$ ) and volume of distribution at steady state $\left(\mathrm{V}_{\mathrm{ss}}\right)$.

In rabbits, data at only three time-points were available, as Nanobody levels were only quantifiable up to $8 \mathrm{~h}$ post-administration. Therefore, a non-compartmental PK analysis (NCA) using Phoenix WinNonlin version 6.3 (Pharsight) was performed on the mean serum concentration-time profile. The area under the curve (AUC) was calculated using the Linear-up-Log-down trapezoidal rule. The terminal phase was determined considering the last two observed time-points only ( $3 \mathrm{~h}$ and $8 \mathrm{~h}$ post-administration).

The nominal dose shown in Table 1, either fixed (Exp. 1-4, $\mu$ g) or body weight adjusted (Exp. 5-8, mg/kg), was used for PK analysis. Nominal sampling times were considered as the actual recorded time was within $5 \%$ of the corresponding nominal time for all bleeds. PK values below the limit of quantification were treated as missing.

\section{Acknowledgments}

The authors would like to greatly acknowledge the following colleagues: Sofie Poelmans and Sigrid Sobry for their work regarding ELISA assay development and PK sample analysis, Stefan De Buck and Alex Hemeryck for their general contribution to the topic of Nanobody pharmacokinetics, Sandy Jacobs and Anne Schoolmeester for their assistance in monitoring the animal studies, Roel Straetemans for critically reviewing the manuscript, Stephanie Staelens, Joachim Boucneau and Pieter Deschaght for Nanobody generation and characterization, and CMC colleagues for test item preparation and characterization.

\section{Author Contributions}

Sven Hoefman, Judith Baumeister and Maria Laura Sargentini-Maier conceived and designed the experiments; Sven Hoefman and Ingrid Ottevaere analyzed the data; Judith Baumeister and Maria Laura Sargentini-Maier contributed reagents/materials/analysis tools; Sven Hoefman, Ingrid Ottevaere and Maria Laura Sargentini-Maier wrote the paper. 


\section{Conflicts of Interest}

Sven Hoefman, Ingrid Ottevaere, Judith Baumeister and Maria Laura Sargentini-Maier are employees of Ablynx NV.

\section{References}

1. Hamers-Casterman, C.; Atarhouch, T.; Muyldermans, S.; Robinson, G.; Hamers, C.; Songa, E.B.; Bendahman, N.; Hamers, R. Naturally occurring antibodies devoid of light chains. Nature 1993, $363,446-448$.

2. Harmsen, M.M.; De Haard, H.J. Properties, production, and applications of camelid single-domain antibody fragments. Appl. Microbiol. Biotechnol. 2007, 77, 13-22.

3. Muyldermans, S. Nanobodies: Natural single-domain antibodies. Ann. Rev. Biochem. 2013, 82, 775-797.

4. Van Bockstaele, F.; Holz, J.-B.; Revets, H. The development of nanobodies for therapeutic applications. Curr. Opin. Investig. Drugs 2009, 10, 1212-1224.

5. Hassanzadeh-Ghassabeh, G.; Devoogdt, N.; De Pauw, P.; Vincke, C.; Muyldermans, S. Nanobodies and their potential applications. Nanomedicine 2013, 8, 1013-1026.

6. Venkatachalam, M.A.; Rennke, H.G. The structural and molecular basis of glomerular filtration. Circul. Res. 1978, 43, 337-347.

7. Lin, J.H. Pharmacokinetics of biotech drugs: peptides, proteins and monoclonal antibodies. Curr. Drug Metab. 2009, 10, 661-691.

8. Rennke, H.G.; Patel, Y.; Venkatachalam, M.A. Glomerular filtration of proteins: Clearance of anionic, neutral, and cationic horseradish peroxidase in the rat. Kidney Int. 1978, 13, 278-288.

9. Peters, T., Jr. Serum albumin. Adv. Protein Chem. 1985, 37, 161-245.

10. Tojo, A.; Endou, H. Intrarenal handling of proteins in rats using fractional micropuncture technique. Am. J. Physiol. 1992, 263, F601-606.

11. Tojo, A.; Kinugasa, S. Mechanisms of glomerular albumin filtration and tubular reabsorption. Int. J. Nephrol. 2012, 2012, Article ID 481520.

12. Ohlson, M.; Sorensson, J.; Haraldsson, B. Glomerular size and charge selectivity in the rat as revealed by FITC-ficoll and albumin. Am. J. Physiol. 2000, 279, F84-F91.

13. Kuwabara, T.; Ishikawa, Y.; Kobayashi, H.; Kobayashi, S.; Sugiyama, Y. Renal clearance of a recombinant granulocyte colony-stimulating factor, nartograstim, in rats. Pharm. Res. 1995, 12, $1466-1469$.

14. Yang, B.B.; Lum, P.K.; Hayashi, M.M.; Roskos, L.K. Polyethylene glycol modification of filgrastim results in decreased renal clearance of the protein in rats. J. Pharm. Sci. 2004, 93, 1367-1373.

15. Kuo, T.T.; Baker, K.; Yoshida, M.; Qiao, S.W.; Aveson, V.G.; Lencer, W.I.; Blumberg, R.S. Neonatal Fc receptor: From immunity to therapeutics. J. Clin. Immunol. 2010, 30, 777-789.

16. Andersen, J.T.; Sandlie, I. The versatile MHC class I-related FcRn protects IgG and albumin from degradation: implications for development of new diagnostics and therapeutics. Drug Metab. Pharmacok. 2009, 24, 318-332. 
17. Sand, K.M.; Bern, M.; Nilsen, J.; Noordzij, H.T.; Sandlie, I.; Andersen, J.T. Unraveling the Interaction between FcRn and Albumin: Opportunities for Design of Albumin-Based Therapeutics. Front. Immunol. 2014, 5, 682.

18. Berson, S.A.; Yalow, R.S.; Schreiber, S.S.; Post, J. Tracer experiments with 1131 labeled human serum albumin: distribution and degradation studies. J. Clin. Invest. 1953, 32, 746-768.

19. Morell, A.; Terry, W.D.; Waldmann, T.A. Metabolic properties of IgG subclasses in man. J. Clin. Invest. 1970, 49, 673-680.

20. Duttaroy, A.; Kanakaraj, P.; Osborn, B.L.; Schneider, H.; Pickeral, O.K.; Chen, C.; Zhang, G.; Kaithamana, S.; Singh, M.; Schulingkamp, R.; et al. Development of a long-acting insulin analog using albumin fusion technology. Diabetes 2005, 54, 251-258.

21. Jazayeri, J.A.; Carroll, G.J. Fc-based cytokines: Prospects for engineering superior therapeutics. BioDrugs 2008, 22, 11-26.

22. Bailon, P.; Won, C.Y. PEG-modified biopharmaceuticals. Exp. Opin. Drug Deliv. 2009, 6, 1-16.

23. Andersen, J.T.; Pehrson, R.; Tolmachev, V.; Daba, M.B.; Abrahmsen, L.; Ekblad, C. Extending half-life by indirect targeting of the neonatal $\mathrm{Fc}$ receptor $(\mathrm{FcRn})$ using a minimal albumin binding domain. J. Biol. Chem. 2011, 286, 5234-5241.

24. Holt, L.J.; Basran, A.; Jones, K.; Chorlton, J.; Jespers, L.S.; Brewis, N.D.; Tomlinson, I.M. Anti-serum albumin domain antibodies for extending the half-lives of short lived drugs. Protein Eng. Des. Sel. 2008, 21, 283-288.

25. Muller, M.R.; Saunders, K.; Grace, C.; Jin, M.; Piche-Nicholas, N.; Steven, J.; O'Dwyer, R.; Wu, L.; Khetemenee, L.; Vugmeyster, Y.; et al. Improving the pharmacokinetic properties of biologics by fusion to an anti-HSA shark VNAR domain. mAbs 2012, 4, 673-685.

26. Tijink, B.M.; Laeremans, T.; Budde, M.; Stigter-van Walsum, M.; Dreier, T.; de Haard, H.J.; Leemans, C.R.; van Dongen, G.A. Improved tumor targeting of anti-epidermal growth factor receptor Nanobodies through albumin binding: Taking advantage of modular Nanobody technology. Mol. Cancer Therap. 2008, 7, 2288-2297.

27. Carter, D.C.; Ho, J.X. Structure of serum albumin. Adv. Protein Chem. 1994, 45, 153-203.

28. Ulrichts, H.; Silence, K.; Schoolmeester, A.; de Jaegere, P.; Rossenu, S.; Roodt, J.; Priem, S.; Lauwereys, M.; Casteels, P.; Van Bockstaele, F.; et al. Antithrombotic drug candidate ALX-0081 shows superior preclinical efficacy and safety compared with currently marketed antiplatelet drugs. Blood 2011, 118, 757-765.

29. Vosjan, M.J.; Perk, L.R.; Roovers, R.C.; Visser, G.W.; Stigter-van Walsum, M.; van Bergen En Henegouwen, P.M.; van Dongen, G.A. Facile labelling of an anti-epidermal growth factor receptor Nanobody with $68 \mathrm{Ga}$ via a novel bifunctional desferal chelate for immuno-PET. Eur. J. Nucl. Med. Mol. Imag. 2011, 38, 753-763.

30. Gainkam, L.O.; Huang, L.; Caveliers, V.; Keyaerts, M.; Hernot, S.; Vaneycken, I.; Vanhove, C.; Revets, H.; De Baetselier, P.; Lahoutte, T. Comparison of the biodistribution and tumor targeting of two 99mTc-labeled anti-EGFR nanobodies in mice, using pinhole SPECT/micro-CT. J. Nucl. Med. 2008, 49, 788-795.

31. Davies, B.; Morris, T. Physiological Parameters in Laboratory Animals and Humans. Pharm. Res. 1993, 10, 1093-1096.

32. Lote, C. Principles of Renal Physiology, 5th ed.; Springer: New York, NY, USA, 2012. 
33. Lenting, P.J.; Westein, E.; Terraube, V.; Ribba, A.S.; Huizinga, E.G.; Meyer, D.; de Groot, P.G.; Denis, C.V. An experimental model to study the in vivo survival of von Willebrand factor. Basic aspects and application to the R1205H mutation. J. Biol. Chem. 2004, 279, 12102-12109.

34. Sarav, M.; Wang, Y.; Hack, B.K.; Chang, A.; Jensen, M.; Bao, L.; Quigg, R.J. Renal FcRn reclaims albumin but facilitates elimination of IgG. J. Am. Soc. Nephrol. 2009, 20, 1941-1952.

35. Kim, J.; Bronson, C.L.; Hayton, W.L.; Radmacher, M.D.; Roopenian, D.C.; Robinson, J.M.; Anderson, C.L. Albumin turnover: FcRn-mediated recycling saves as much albumin from degradation as the liver produces. Am. J. Physiol. 2006, 290, G352-G360.

36. Borvak, J.; Richardson, J.; Medesan, C.; Antohe, F.; Radu, C.; Simionescu, M.; Ghetie, V.; Ward, E.S. Functional expression of the MHC class I-related receptor, FcRn, in endothelial cells of mice. Int. Immunol. 1998, 10, 1289-1298.

37. Nguyen, A.; Reyes, A.E., 2nd; Zhang, M.; McDonald, P.; Wong, W.L.; Damico, L.A.; Dennis, M.S. The pharmacokinetics of an albumin-binding Fab (AB.Fab) can be modulated as a function of affinity for albumin. Protein Eng. Des. Sel. 2006, 19, 291-297.

38. Lobo, E.D.; Hansen, R.J.; Balthasar, J.P. Antibody pharmacokinetics and pharmacodynamics. J. Pharm. Sci. 2004, 93, 2645-2668.

39. Deng, R.; Iyer, S.; Theil, F.P.; Mortensen, D.L.; Fielder, P.J.; Prabhu, S. Projecting human pharmacokinetics of therapeutic antibodies from nonclinical data: What have we learned? $m A b s$ 2011, 3, 61-66.

40. Petkova, S.B.; Akilesh, S.; Sproule, T.J.; Christianson, G.J.; Al Khabbaz, H.; Brown, A.C.; Presta, L.G.; Meng, Y.G.; Roopenian, D.C. Enhanced half-life of genetically engineered human IgG1 antibodies in a humanized FcRn mouse model: potential application in humorally mediated autoimmune disease. Int. Immunol. 2006, 18, 1759-1769.

41. Vugmeyster, Y.; Szklut, P.; Tchistiakova, L.; Abraham, W.; Kasaian, M.; Xu, X. Preclinical pharmacokinetics, interspecies scaling, and tissue distribution of humanized monoclonal anti-IL-13 antibodies with different IL-13 neutralization mechanisms. Int. Immunopharm. 2008, 8, 477-483.

42. Lin, Y.S.; Nguyen, C.; Mendoza, J.L.; Escandon, E.; Fei, D.; Meng, Y.G.; Modi, N.B. Preclinical pharmacokinetics, interspecies scaling, and tissue distribution of a humanized monoclonal antibody against vascular endothelial growth factor. J. Pharm. Exp. Therap. 1999, 288, 371-378.

43. Ober, R.J.; Radu, C.G.; Ghetie, V.; Ward, E.S. Differences in promiscuity for antibody-FcRn interactions across species: implications for therapeutic antibodies. Int. Immunol. 2001, 13, 1551-1559.

44. Holz, J.B. The TITAN trial--assessing the efficacy and safety of an anti-von Willebrand factor Nanobody in patients with acquired thrombotic thrombocytopenic purpura. Transf. Apheresis Sci. 2012, 46, 343-346.

45. De Bruyn, S.; De Smedt, T.; Allosery, K.; Crabbe, P.; De Brabandere, V.; Detalle, L.; Mortier, K.; Schoolmeester, A.; Wouters, A.; Stöhr, T.; et al. ALX-0171: Safety and Therapeutic Potential of an Inhaled Anti-RSV Nanobody. Respirat. Drug Deliv. Eur. 2015, in press.

46. Holz, J.B.; Sargentini-Maier, L.; De Bruyn, S.; Gachályi, B.; Udvaros, I.; Rojkovich, B.; Bruk, S.; Sramek, P.; Korkosz, M.; Krause, K.; et al. Twenty-four weeks of treatment with a novel anti-IL6 receptor nanobody (ALX-0061) resulted in 84\% ACR20 improvement and 58\% DAS28 remission in a phase I/II study in RA. Ann. Rheumat. Dis. 2013, 72, 64. 
47. Arbabi Ghahroudi, M.; Desmyter, A.; Wyns, L.; Hamers, R.; Muyldermans, S. Selection and identification of single domain antibody fragments from camel heavy-chain antibodies. FEBS Lett. 1997, 414, 521-526.

(C) 2015 by the authors; licensee MDPI, Basel, Switzerland. This article is an open access article distributed under the terms and conditions of the Creative Commons Attribution license (http://creativecommons.org/licenses/by/4.0/). 